\title{
Evaluación del rendimiento diagnóstico de un nomograma de predicción de preeclampsia
}

\section{Evaluation of the diagnostic performance of a preeclampsia prediction nomogram}

\section{Claudia M. López-Jurado1*, Dante M. Quiñones-Laveriano² y Jhony De la Cruz-Vargas²}

${ }^{1}$ Facultad de Medicina Humana "Manuel Huamán Guerrero"; 2 Instituto de Investigación en Ciencias Biomédicas. Universidad Ricardo Palma, Lima, Perú

\section{Resumen}

Objetivo: Determinar el rendimiento diagnóstico de un nomograma de predicción de preeclampsia en gestantes atendidas en el Hospital Nacional Dos de Mayo en Lima, Perú de enero de 2016 a julio de 2019. Método: Estudio de tipo pruebas diagnósticas. Se contó con una muestra de 513 pacientes; de ellas, 225 con diagnóstico de preeclampsia y 288 sin dicho diagnóstico. El análisis de los datos obtenidos se realizó en STATA v.14 y se determinaron la sensibilidad, la especificidad, el valor predictivo positivo y el valor predictivo negativo. Resultados: Se analizó la curva ROC y se encontró un área bajo la curva de 0,91 (intervalo de confianza del 95\%: 0,89-0,93), obteniendo 187 puntos como valor de corte de mejor rendimiento diagnóstico, con una sensibilidad del $81,33 \%$ y una especificidad del $85,76 \%$. Conclusiones: El nomograma de predicción de preeclampsia tiene un buen rendimiento diagnóstico para las gestantes atendidas en el servicio de ginecología y obstetricia del Hospital Nacional Dos de Mayo.

Palabras clave: Preeclampsia. Predicción. Toxemias del embarazo.

\section{Abstract}

Objective: Determine the diagnostic performance of a preeclampsia prediction nomogram in pregnant women attended at the Dos de Mayo National Hospital during the period from January 2016 to July 2019. Method: Study of diagnostic tests. There was a sample of 513 patients, 225 patients with a diagnosis of pre-eclampsia and 288 patients without such diagnosis were selected. The analysis of the data obtained was carried out in STATA v.14, obtaining the values of sensitivity, specificity, PPV and NPV. Results: The ROC curve was analyzed obtaining an AUC of 0.91 (95\% Cl: 0.89 - 0.93), obtaining 187 points as the cut-off point for the best diagnostic performance, with a sensitivity of $81.33 \%$ and a specificity of $85.76 \%$. Conclusions: The preeclampsia prediction nomogram has a good diagnostic performance for pregnant women attended at the Gynecology and Obstetrics service of the Dos de Mayo National Hospital.

Key words: Pre-eclampsia. Prediction. Toxemia pregnancy.

Disponible en internet: 02-08-2021 Rev Chil Obstet Ginecol. 2021;86(3):282-290

www. rechog.com 0048-766X / @ 2021 Sociedad Chilena de Obstetricia y Ginecología. Publicado por Permanyer. Éste es un artículo open access bajo la licencia CC BY-NC-ND (https://creativecommons.org/licenses/by-nc-nd/4.0/). 


\section{Introducción}

La preeclampsia constituye un problema de salud pública, es la segunda causa de muerte materna en el mundo, con un $14 \%$, y se ha estimado que complica un 2-8\% de los embarazos en el mundo. En Perú, en 2018, se reportó como la primera causa de muerte materna, con $28,7 \%$. Por ello, es importante identificar tempranamente a las mujeres con alta predisposición para desarrollar preeclampsia.

Diversos estudios han planteado el uso de nomogramas predictores, pero pocos reportan resultados prometedores debido a la heterogeneidad de sus variables, y parecen ser beneficiosos para una población con características particulares, ya que al realizar validaciones externas en poblaciones diferentes de las que originalmente se diseñaron se obtuvo un peor rendimiento. Esto es especialmente cierto en contextos de bajos recursos, como el peruano, donde no se ha podido determinar aún el mejor instrumento para este fin².

Para identificar gestantes con alto riesgo de preeclampsia es necesaria la combinación de características clínicas, marcadores séricos y pruebas ecográficas. Sin embargo, el uso de marcadores séricos específicos, como el factor de crecimiento placentario y la proteína plasmática A asociada al embarazo (PAPP-A), es poco accesible.

En Perú existe un nomograma de predicción de preeclampsia, diseñado por Alegría y Gonzales ${ }^{3}$ entre los años 2014 y 2015 para gestantes atendidas en un hospital del Seguro Social de Salud (EsSalud) en Lima, Perú, teniendo alta sensibilidad (S) y buena especificidad $(E)$, usando indicadores accesibles en un hospital de baja complejidad. La población asegurada en EsSalud tiene características socioeconómicas y educativas diferentes de la mayoría de la población peruana ${ }^{4}$, la cual está asegurada principalmente en el Seguro Integral de Salud (SIS). Siendo que estos factores pueden influir en el desarrollo de preeclampsia ${ }^{5,6}$, surge la necesidad de evaluar este nomograma en una población beneficiaria, en su mayoría, del SIS, como son las gestantes atendidas en el servicio de ginecología y obstetricia del Hospital Nacional Dos de Mayo (HNDM). Por ello, el objetivo de nuestro estudio fue determinar el rendimiento diagnóstico de un nomograma de predicción de preeclampsia en gestantes atendidas en el HNDM durante el periodo de enero de 2016 a julio de 2019.

\section{Método}

\section{Diseño del estudio}

El presente estudio es de pruebas diagnósticas.

\section{Población}

La población estuvo constituida por todas las gestantes atendidas en el servicio de ginecología y obstetricia del HNDM durante el periodo de enero de 2016 a julio de 2019, con y sin diagnóstico de preeclampsia. Se incluyeron gestantes entre 15 y 55 años que contaran con los datos clínicos y auxiliares de interés del trabajo descritos en sus historias clínicas. Se excluyeron las gestantes con historias clínicas incompletas, sin diagnóstico de ingreso definitivo, las que tuvieron como resultado un aborto, amenaza de aborto o feto con malformaciones congénitas, y aquellas a quienes se le realizó cesárea de emergencia sin diagnóstico establecido.

\section{Muestreo}

Las pacientes registradas fueron de 241 gestantes con preeclampsia, de las cuales se excluyó a 16 pacientes por no cumplir con los criterios de inclusión. El total del pacientes incluidas en el estudio fue de 225 gestantes con preeclampsia. Se decidió incluir a toda la población para las pacientes sin diagnóstico de preeclampsia mediante un muestreo aleatorio simple a partir de los registros de las historias clínicas durante el periodo de estudio.

\section{Tamaño muestral}

Se usó la calculadora EPIDAT-SERGAS, versión 4.2, y haciendo uso de los valores establecidos en el estudio de Alegría y Gonzales ${ }^{3}$ se utilizaron una $S$ del $82,2 \%$, una $\mathrm{E}$ del $75,1 \%$ y un nivel de confianza del $95 \%$. Con una precisión del $5 \%$ se obtuvo una muestra de 225 mujeres con presencia de la enfermedad y 288 sin presencia de la enfermedad.

\section{Procedimientos}

La técnica de recolección de datos fue mediante revisión de las historias clínicas. Se usó una ficha de recolección de datos en la que se registraron las variables de importancia para el estudio.

Se presentó el protocolo de investigación al comité de ética de la Facultad de Medicina Humana de la 
Universidad Ricardo Palma para su aprobación, y posteriormente fue enviado al comité de ética del HNDM también para su aprobación, donde fue otorgado el permiso para la revisión de las historias clínicas.

Se tuvo acceso al registro y se filtró a todas las pacientes con diagnóstico de preeclampsia (CIE10: O14), siendo 241 pacientes las que integraron el grupo de casos. En el resto del registro quedaron 8747 pacientes sin diagnóstico de preeclampsia, de las que se escogieron 288 para el grupo control mediante un muestreo aleatorio simple. Seguidamente, se acudió a la Oficina de Archivos e Historias clínicas, en donde se realizó una revisión de las historias clínicas. Hecho esto, los datos fueron ingresados a una matriz de datos de Microsoft Excel $囚$, en la que se ordenaron y se asignaron los puntajes establecidos según el nomograma de predicción de preeclampsia de Alegría y Gonzales ${ }^{3}$, determinando en qué categoría de riesgo se encontraba cada paciente, con lo cual se evaluó si este test predijo o no el desarrollo del evento de estudio, siendo necesario dicotomizar las categorías mencionadas en bajo riesgo (bajo) y alto riesgo (intermedio y alto) para luego evaluar la $\mathrm{S}$, la $\mathrm{E}$, el valor predictivo positivo (VPP) y el valor predictivo negativo (VPN).

Los datos obtenidos fueron los del primer control prenatal o de la primera vez que se registró el dato previsto, siendo en todos los casos no mayor de 20 semanas de gestación. El índice de pulsatilidad promedio de las arterias uterinas se obtuvo a partir del promedio de los índices de pulsatilidad de las arterias uterinas izquierda y derecha, logrados mediante ecografía Doppler. Las arterias uterinas fueron identificadas en un plano oblicuo de la pelvis en el cruce con las arterias hipogástricas de cada lado. La muestra se tomó cuando se encontraron tres ondas consecutivas similares. Se registró el percentil en el que se ubicaba el índice de pulsatilidad promedio de las arterias uterinas según un estudio previo?.

\section{Variables a medir}

El evento de interés fue la preeclampsia, considerándose a las pacientes de al menos 20 semanas de gestación con presión arterial sistólica $\geq 140 \mathrm{mmHg}$ o diastólica $\geq 90 \mathrm{mmHg}$ y que previamente tuvieran una presión arterial normal, acompañado de proteinuria, evidencia de insuficiencia orgánica o síntomas premonitorios $^{8}$. Para predecir esta variable se utilizó el nomograma de predicción de preeclampsia de Alegría y Gonzales ${ }^{3}$, el cual fue diseñado y evaluado en un estudio de casos y controles anidados con una razón de 3:1, siendo 119
Tabla 1. Variables incluidas en el nomograma de Alegría y Gonzales

\section{Paridad}

Antecedente personal de preeclampsia 0 en primera línea

Edad materna$$
\text { (1) }
$$

(n)

\section{Presión arterial media}

Índice de pulsatilidad promedio de las arterias uterinas

teinuria en 24 horas

Presencia de comorbilidad 0 alguna condición médica
Nulípara: 20 puntos Multípara: 5 puntos

Sí: 25 puntos No: 0 puntos

$\leq 15$ años: 10 puntos 16 a 24 años: 13 puntos 25 a 29 años: 16 puntos 30 a 34 años: 19 puntos 35 a 39 años: 22 puntos $\geq 40$ años: 25 puntos

$\leq 70 \mathrm{mmHg}: 0$ puntos 71 a $75 \mathrm{mmHg}: 5$ puntos 76 a $80 \mathrm{mmHg}: 10$ puntos 81 a $85 \mathrm{mmHg}: 17,5$ puntos 86 a $90 \mathrm{mmHg}: 25$ puntos 91 a $95 \mathrm{mmHg}: 32,5$ puntos 96 a $100 \mathrm{mmHg}$ : 40 puntos 101 a $105 \mathrm{mmHg}$ : 47,5 puntos 106 a $110 \mathrm{mmHg}$ : 55 puntos

$15 \mathrm{~kg} / \mathrm{m}^{2}: 25$ puntos $20 \mathrm{~kg} / \mathrm{m}^{2}: 40$ puntos $25 \mathrm{~kg} / \mathrm{m}^{2}: 55$ puntos $30 \mathrm{~kg} / \mathrm{m}^{2}: 70$ puntos $35 \mathrm{~kg} / \mathrm{m}^{2}: 85$ puntos $40 \mathrm{~kg} / \mathrm{m}^{2}: 100$ puntos

Percentil 50: 30 puntos Percentiles 50 a 54: 35 puntos Percentiles 60 a 64: 45 puntos Percentiles 70 a $74: 55$ puntos Percentiles 80 a 84: 65 puntos Percentiles 90 a 94: 75 puntos Percentiles $\geq 95$ : 85 puntos

150 a $159 \mathrm{mg} / 24 \mathrm{~h}: 20$ puntos 200 a $209 \mathrm{mg} / 24 \mathrm{~h}: 35$ puntos 250 a $259 \mathrm{mg} / 24 \mathrm{~h}: 50$ puntos 300 a $309 \mathrm{mg} / 24 \mathrm{~h}: 65$ puntos 350 a $359 \mathrm{mg} / 24 \mathrm{~h}: 80$ puntos $\geq 400 \mathrm{mg} / 24 \mathrm{~h}: 95$ puntos

Sí: 10 puntos No: 0 puntos los casos y 357 los controles, en gestantes atendidas en el Hospital Nacional Guillermo Almenara Irigoyen entre el primer y el segundo trimestre de embarazo, obteniendo una $S$ del $82,2 \%$, una $E$ del $75,1 \%$, un VPN del $86,6 \%$ y un VPP del $78,8 \%$. El nomograma está conformado por las variables mostradas en la tabla 1.

\section{Análisis estadístico}

Se elaboró una base de datos en el programa STATA versión 14. Para el análisis de las variables cualitativas 


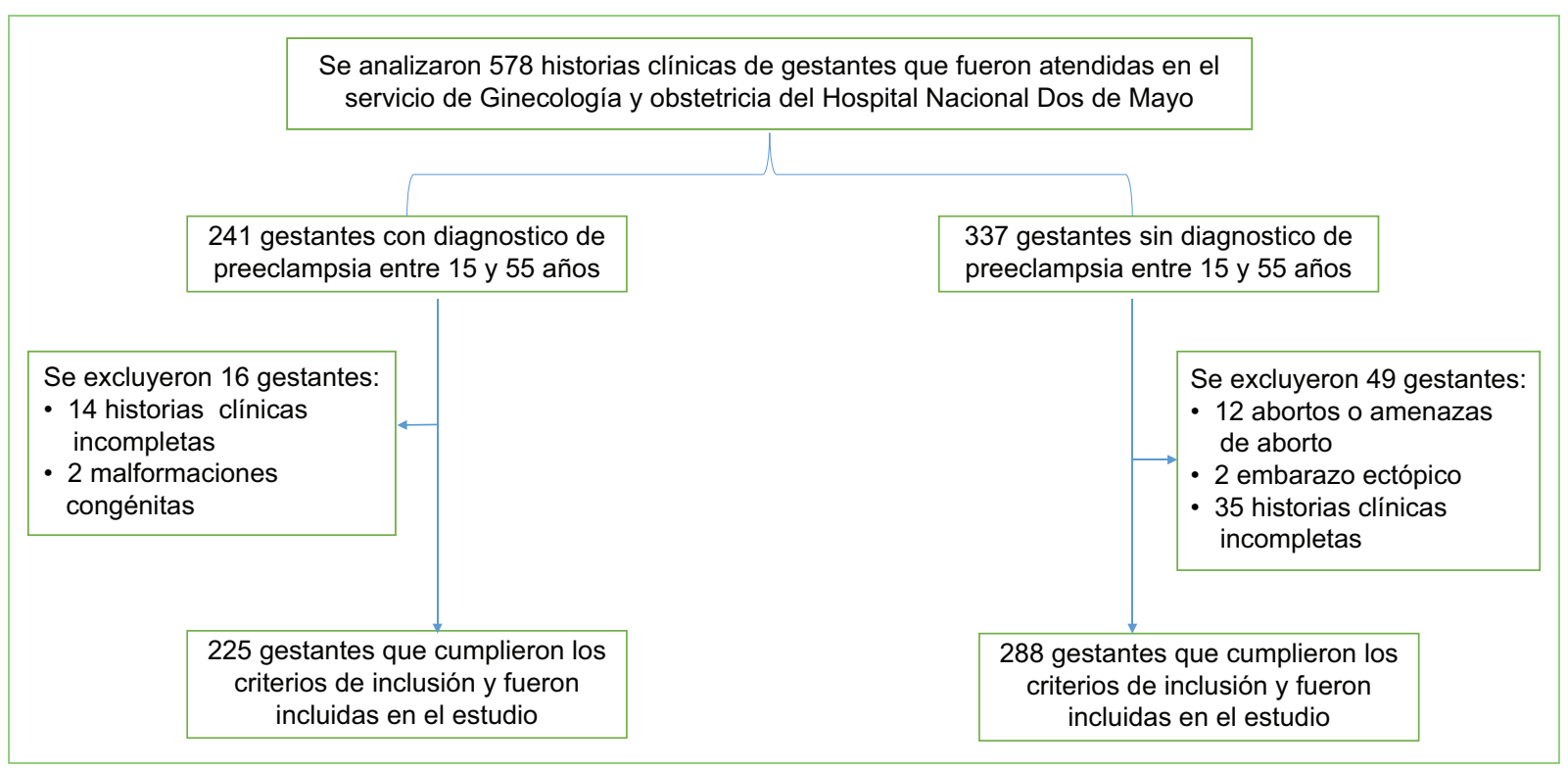

Figura 1. Flujograma de participantes incluidas en el estudio.

se determinaron las frecuencias absolutas y relativas (porcentajes), y para las variables cuantitativas se determinaron medidas de tendencia central (promedio) y medidas de dispersión (desviación estándar). Se estableció el valor diagnóstico del nomograma mediante el análisis de la curva ROC (Receiver Operating Characteristic) para cada punto de corte y se escogió el que tenía mayores $\mathrm{S}$ y $\mathrm{E}$. Se analizaron el VPP y el VPN para determinar la seguridad de esta prueba diagnóstica. Los resultados obtenidos según el puntaje final se agruparon en bajo riesgo (0-186 puntos) y alto riesgo ( $\geq 187$ puntos), siendo este corte el de mayor rendimiento diagnóstico.

\section{Consideraciones éticas}

El presente estudio no trabajó directamente con las gestantes ni realizó ningún tipo de procedimiento, por lo que no constituyó ningún riesgo para las participantes. Los datos fueron manejados únicamente por los autores, codificando cualquier información que permitiera la identificación de las pacientes. El proyecto de investigación fue revisado por el Comité de Ética de la Universidad Ricardo Palma, que autorizó su realización (PG-20-2020).

\section{Resultados}

Se analizaron 578 historias clínicas, de las cuales se descartaron 65 porque no contenían los datos necesarios para ser incluidas en el estudio, quedando para el análisis 513 pacientes (Fig. 1). De estas, la mediana de la edad fue de 26 años (Rango intercuatilico: 22-32). En cuanto a la paridad, el total de nulíparas fue de $274(53,4 \%)$ y el total de multíparas fue de $239(46,5 \%)$. El antecedente de preeclampsia en un embarazo previo o de primera línea se presentó en $100(19,5 \%)$, y en quienes no estuvo presente fueron $413(80,5 \%)$. Las características generales de la población se pueden observar en la tabla 2.

Se analizaron la comorbilidad y las condiciones médicas de la población, siendo lo más frecuente antecedentes familiares de hipertensión arterial (HTA), con 56 (53,8\%), seguida de HTA crónica, con 12 (11,6\%). La comorbilidad y las condiciones médicas de la población se muestran en la figura 2.

El análisis de la curva ROC (Fig. 3) del puntaje del nomograma de predicción de preeclampsia arrojó un área bajo la curva (AUC) de 0,91 (intervalo de confianza del 95\% [IC95\%]: 0,89-0,93). El punto de corte de mejor rendimiento diagnóstico fue de 187 puntos, con una $S$ del $81,33 \%$ y una $E$ del $85,76 \%$, obteniendo el punto de corte con mayor sensibilidad con 174 puntos.

El puntaje con mejor rendimiento en el estudio fue 187 puntos, seguido de los puntos de corte propuestos en el estudio original de Alegría y Gonzales ${ }^{3}$, en el cual 190 puntos establecían un bajo riesgo, 230 puntos un riesgo intermedio y 320 puntos un alto riesgo de desarrollar preeclampsia. 
Tabla 2. Características generales de las gestantes atendidas en el Hospital Nacional Dos de Mayo durante el periodo de enero de 2016 a julio de 2019

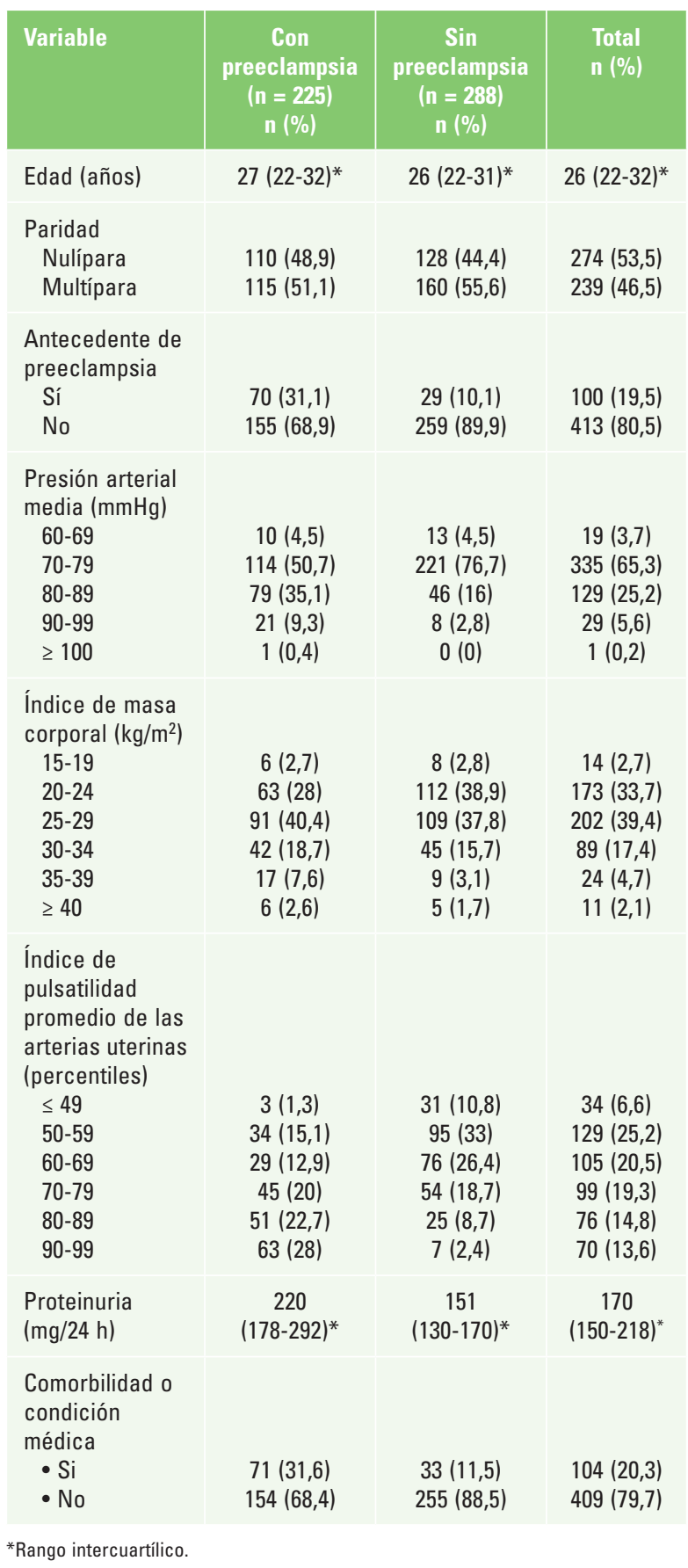

El rendimiento diagnóstico de estos puntos de corte, así como los propuestos en el estudio original del nomograma evaluado, se exponen en la tabla 3.

En la tabla 4 se detallan los valores del AUC de cada variable, siendo los que tuvieron mayor AUC la proteinuria de 24 horas,con un AUC de 0,87 (IC95\%: 0,84$0,90)$, y el índice de pulsatilidad promedio de las arterias uterinas, con un AUC de 0,76 (IC95\%: 0,720,80 ); los que tuvieron menor AUC fueron la paridad, con un AUC de 0,52 (IC95\%: 0,48-0,57), y la edad materna, con un AUC de 0,52 (IC95\%: 0,47-0,57).

\section{Discusión}

La preeclampsia es una de las patologías del embarazo que causa mayor morbimortalidad tanto materna como perinatal ${ }^{1,9}$. A pesar de los avances, el único tratamiento definitivo es la culminación del embarazo; empero, mediante la prevención precoz se puede reducir su carga ${ }^{10}$. El American College of Obstetricians and Gynecologists recomienda el uso de ácido acetilsalicílico para prevenirla, ya que puede reducir el riesgo de presentarla y sus complicaciones perinatales ${ }^{11,12}$. A su vez, la última guía de manejo de preeclampsia en el Perú aconseja el uso de ácido acetilsalicílico en gestantes con riesgo de desarrollar preeclampsia, desde las 14 hasta las 34 semanas de gestación ${ }^{13}$. Sin embargo, en la práctica diaria no se suele recetar, pues no se cuenta con un tamizaje adecuado para identificar pacientes con riesgo de preeclampsia.

Las investigaciones previas han dado a conocer que la nuliparidad ${ }^{14}$, el antecedente de preeclampsia ${ }^{14-16}$, el antecedente de hipertensión arterial crónica ${ }^{6,13}$, las enfermedades autoinmunitarias ${ }^{16,17}$, las edades extremas $^{6,15,18}$, el índice de masa corporal (IMC) alto ${ }^{16,19,20}$, la elevación de la presión arterial media (PAM) ${ }^{21,22}$, el índice de pulsatilidad de las arterias uterinas ${ }^{20,22-25}$ y los valores aumentados de proteinuria ${ }^{10,25}$ estarían asociados al desarrollo de preeclampsia. Por ello, se analizó el AUC de cada variable, resultando que la proteinuria en 24 horas mostraba una de las mayores AUC, con 0,87, cercana al valor encontrado en el estudio de Alegría y Gonzales ${ }^{3}$, en el cual se obtuvo un AUC de 0,96; en ambos casos se observó un buen rendimiento, lo cual podría deberse a que esta variable estaba relacionada con los criterios diagnósticos de la enfermedad ${ }^{26}$. El índice de pulsatilidad promedio de las arterias uterinas obtuvo un AUC de 0,76, a diferencia del estudio de Alegría y Gonzales ${ }^{3}$, en el que obtuvieron un AUC de 0,95; esto podría deberse a que la patogénesis de la enfermedad se caracteriza por una pobre circulación uteroplacentaria, resultando en una hipoperfusión placentaria, lo que llevaría a infartos placentarios, hipoplasia vellosa e isquemia ${ }^{22,27}$, todo esto desencadenando la preeclampsia.

Espinoza Villena ${ }^{28}$ elaboró y evaluó un nomograma de predicción de preeclampsia, obteniendo un AUC de 0,76 (IC95\%: 0,65-0,781), una $S$ del $79,7 \%$ y una $E$ del 


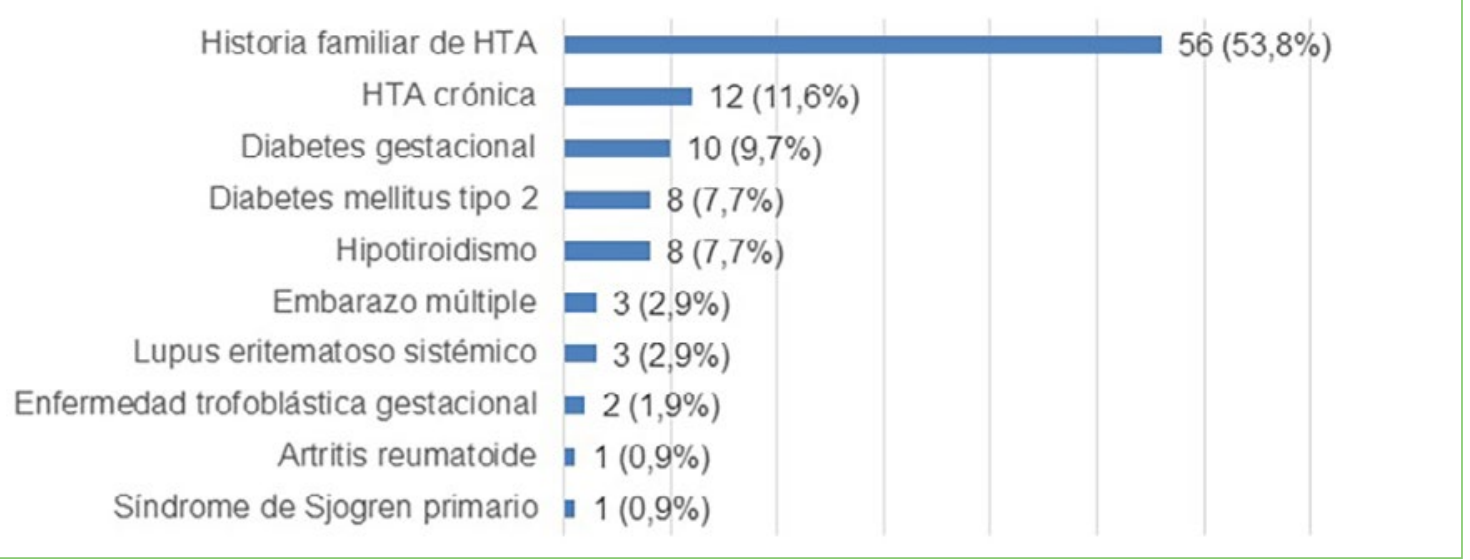

Figura 2. Comorbilidad o condiciones médicas de las gestantes atendidas en el Hospital Nacional Dos de Mayo durante el periodo de enero de 2016 a julio de 2019. HTA: hipertensión arterial.

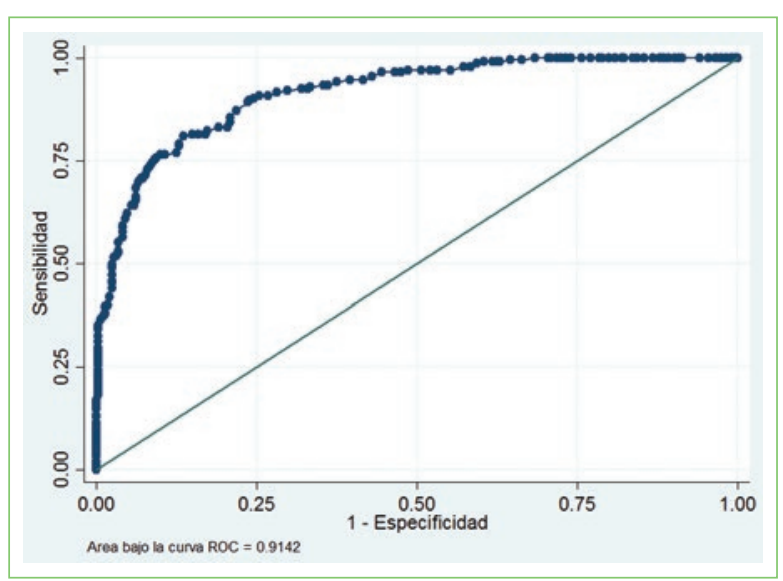

Figura 3. Curva ROC del puntaje obtenido por el nomograma de predicción de preeclampsia de Alegría y Gonzales ${ }^{3}$ en las gestantes atendidas en el Hospital Nacional Dos de Mayo durante el periodo de enero de 2016 a julio de 2019.

$53,8 \%$, teniendo un rendimiento moderado, lo cual puede deberse a que su estudio solo incluía variables clínicas (edad materna, paridad, antecedente de embarazo múltiple o preeclampsia), a diferencia del nomograma usado en el presente estudio, en el cual, además de las variables mencionadas, se incluyeron otras variables clínicas, como la PAM y el IMC, y exámenes auxiliares, como el índice de pulsatilidad promedio de las arterias uterinas y la proteinuria en 24 horas, lo cual podría mejorar el desempeño del nomograma y, por tanto, tener un mejor rendimiento.

A su vez, Goetzinger et al. ${ }^{29}$ desarrollaron un sistema de puntuación para predecir la preeclampsia y obtuvieron un AUC de 0,78 (IC95\%: 0,69-0,86), una $S$ del
$36,7 \%$ y una $E$ del $93,2 \%$. Incluyeron variables clínicas (antecedente de HTA crónica o preeclampsia, diabetes gestacional, IMC $>30 \mathrm{~kg} / \mathrm{m}^{2}$ ) y exámenes auxiliares (PAPP-A y muesca diastólica de arteriales uterinas), lo cual se diferencia del presente estudio, ya que este incluye otras variables, como antecedente de preeclampsia en primera línea, índice de pulsatilidad promedio de las arterias uterinas, proteinuria en 24 horas y presencia de alguna condición médica 0 comorbilidad, razón por la cual se podría deducir que nuestro estudio puede haber tenido un mejor rendimiento en cuanto a mayor identificación de casos de preeclampsia.

Por otro lado, Díaz Cobos ${ }^{30}$ realizó una validación externa de un modelo de predicción de preeclampsia, obteniendo un AUC de 0,69 (IC95\%: 0,45-0,93), utilizando variables como la edad materna, el IMC, la etnia, los antecedentes patológicos (diabetes mellitus, HTA, preeclampsia, nefropatías o trombofilias), la paridad, la PAM, el índice de pulsatilidad promedio de las arterias uterinas y la PAPP-A. Een el presente estudio, además, se incluyeron variables como la proteinuria en 24 horas y otra comorbilidad o condiciones médicas, lo cual podría determinar una mayor captación de pacientes y aumentaría el rendimiento del AUC.

El estudio de Alegría y Gonzalez ${ }^{3}$ incluyó 119 casos y 357 controles, a diferencia del presente estudio, que contó con 225 casos y 288 controles. También existen diferencias significativas en cuanto a la presencia de comorbilidad o condiciones médicas que presentan las gestantes estudiadas, como se ha descrito en estudios peruanos $^{31,32}$. La accesibilidad a los servicios de salud está determinada por factores sociales como la 
Tabla 3. Rendimiento predictivo de los diferentes puntos de corte propuestos en el nomograma evaluado en las gestantes atendidas en el Hospital Nacional Dos de Mayo durante el periodo de enero de 2016 a julio de 2019

\begin{tabular}{|c|c|c|c|c|c|c|c|c|}
\hline \multirow[t]{2}{*}{ Punto de corte } & \multicolumn{2}{|c|}{ Preeclampsia } & \multirow[t]{2}{*}{ S } & \multirow[t]{2}{*}{ E } & \multirow[t]{2}{*}{ VPP } & \multirow[t]{2}{*}{ VPN } & \multirow[t]{2}{*}{ LRt } & \multirow[t]{2}{*}{ LR- } \\
\hline & Sí & No & & & & & & \\
\hline $\begin{array}{l}187 \\
\text { Con riesgo } \\
\text { Sin riesgo }\end{array}$ & $\begin{array}{c}183 \\
42\end{array}$ & $\begin{array}{c}41 \\
247\end{array}$ & $80,89 \%$ & $86,46 \%$ & $81,69 \%$ & $85,46 \%$ & 5,97 & 0,22 \\
\hline $\begin{array}{l}190 \\
\text { Con riesgo } \\
\text { Sin riesgo }\end{array}$ & $\begin{array}{c}174 \\
51\end{array}$ & $\begin{array}{c}39 \\
249\end{array}$ & $76,89 \%$ & $87,50 \%$ & $81,69 \%$ & $83 \%$ & 6,15 & 0,26 \\
\hline $\begin{array}{l}230 \\
\text { Con riesgo } \\
\text { Sin riesgo }\end{array}$ & $\begin{array}{c}83 \\
142\end{array}$ & $\begin{array}{c}2 \\
286\end{array}$ & $36,88 \%$ & $99,13 \%$ & $97,64 \%$ & $66,58 \%$ & 44,15 & 0,64 \\
\hline $\begin{array}{l}320 \\
\text { Con riesgo } \\
\text { Sin riesgo }\end{array}$ & $\begin{array}{c}4 \\
221\end{array}$ & $\begin{array}{c}0 \\
288\end{array}$ & $1,78 \%$ & $100 \%$ & $100 \%$ & $56,58 \%$ & - & 0,98 \\
\hline
\end{tabular}

E: especificidad; LR+: likelihood ratio +; LR-: likelihood ratio -; S: sensibilidad; VPN: valor predictivo negativo; VPP: valor predictivo positivo.

Tabla 4. Área bajo la curva de las variables consideradas en el nomograma evaluado en las gestantes atendidas en el Hospital Nacional Dos de Mayo durante el periodo de enero de 2016 a julio de 2019

\begin{tabular}{|l|c|c|c|c|}
\hline Parámetros & AUC & Desviación estándar & \multicolumn{2}{c}{ IC95 \% } \\
\cline { 1 - 2 } & & & Límite inferior & 0,57 \\
\hline Paridad & 0,52 & 0,02 & 0,48 & 0,64 \\
\hline Antecedente de preeclampsia & 0,60 & 0,02 & 0,57 & 0,57 \\
\hline Edad materna & 0,52 & 0,02 & 0,47 & 0,73 \\
\hline Presión arterial media & 0,69 & 0,02 & 0,64 & 0,63 \\
\hline Índice de masa corporal & 0,58 & 0,02 & 0,53 & 0,80 \\
\hline Índice de pulsatilidad promedio de las arterias uterinas & 0,76 & 0,02 & 0,72 & 0,90 \\
\hline Proteinuria en 24 horas & 0,87 & 0,01 & 0,84 & 0,64
\end{tabular}

AUC: área bajo la curva; IC95\%: intervalo de confianza del $95 \%$.

educación, el hacinamiento y la alimentación, siendo que la población asegurada al SIS (que constituye la mayor parte de las pacientes que acuden al HNDM) en su gran mayoría solo han completado el nivel de secundaria, viven en condiciones de hacinamiento y su alimentación se basa en hidratos de carbono, a diferencia de la población con mayor poder adquisitivo que pertenece al EsSalud o a centros de salud privados, esto también determinado por factores económicos, ya que cuentan con un ingreso mensual superior a s/.1538 y cubren sus necesidades médicas. Por otro lado, el estudio de Benites-Zapata et al. $^{4}$ afirma que las personas afiliadas al SIS tienen mayor prevalencia de no utilización de los servicios médicos que las personas afiliadas a EsSalud, recurriendo a agentes de la comunidad (chamán, curanderos, etc.) ante situaciones que afecten su salud, siendo esta una gran barrera de desinformación y para la toma de decisiones preventivas. Todo ello podría contribuir al desarrollo de patologías prevalentes en esta población, como diabetes mellitus, HTA crónica, endocrinopatías y diabetes gestacional, entre otras. En el presente estudio, la variable comorbilidad obtuvo un AUC de 0,60, mientras que en el estudio de Alegría y Gonzales $^{3}$ fue de 0,39, lo cual 
podría explicar el mejor rendimiento del puntaje en esta población.

En cuanto al rendimiento, se obtuvo un AUC de 0,91 (IC95\%: 0,89-0,93). El mejor puntaje encontrado para predecir preeclampsia fue de 187 puntos, con una $S$ del $81,33 \%$, una E del $85,76 \%$, un VPP del $81,69 \%$ y un VPN del $85,46 \%$. Consecuentemente, dado el buen rendimiento pronóstico, el nomograma evaluado podría funcionar en las gestantes atendidas en el HNDM a manera de cribado para la toma de decisiones respecto a medidas preventivas de preeclampsia.

El estudio tuvo como limitaciones que, en cuanto al valor de la proteinuria en 24 horas, se utilizaron tres diferentes exámenes de laboratorio (proteinuria en 24 horas, proteínas en orina mediante tiras reactivas e índice proteinuria/creatinina tomado de una muestra de orina), haciendo uso de la calculadora virtual NEFROCALC, con la cual se obtuvo el valor final de la proteinuria en 24 horas, lo cual puede sesgar los valores obtenidos en esta variable; sin embargo, no descarta su valor diagnóstico, pues aunque la prueba de orina de 24 horas para la proteína total sigue siendo el método de referencia, tiene sus limitaciones, lo que justificaría el uso de alternativas como la tira reactiva, siendo esta más imple y menos costosa, o el índice proteína/creatinina, que es relativamente más rápido $\mathrm{e}$ igualmente preciso para la detección y la cuantificación de la proteinuria, correlacionándose bien con la estimación de proteínas en orina de 24 horas, y por lo tanto podría usarse como una alternativa a las pruebas de orina de 24 horas para pacientes con sospecha de preeclampsia ${ }^{33}$. Por otro lado, siendo este un estudio retrospectivo, puede tener un sesgo de información, ya que las variables ya han sido recogidas por quien realizó la historia clínica, limitándonos los investigadores a su recopilación; sin embargo, los resultados son viables, ya que la toma de decisiones en estas pacientes se basa en la evidencia de la historia clínica, siendo este un documento médico legal y todos los datos consignados ahí son consignados por profesionales de salud.

\section{Conclusiones}

El nomograma de predicción de preeclampsia tiene un buen rendimiento diagnóstico, buenas $S$ y $\mathrm{E}$, y buenos VPP y VPN, pudiendo considerarse como una herramienta útil para la detección precoz de preeclampsia en las gestantes atendidas en el HNDM. Se recomienda fortalecer la evidencia que permita la recomendación o no del uso de este nomograma como parte de la práctica clínica habitual.

\section{Agradecimientos}

A los médicos del servicio de ginecología y obstetricia del HNDM por su contribución para obtener la base de datos de su servicio.

\section{Financiamiento}

Autofinanciado.

\section{Conflicto de intereses}

Los autores declaran no tener conflictos de intereses.

\section{Responsabilidades éticas}

Protección de personas y animales. Los autores declaran que para esta investigación no se han realizado experimentos en seres humanos ni en animales.

Confidencialidad de los datos. Los autores declaran que han seguido los protocolos de su centro de trabajo sobre la publicación de datos de pacientes.

Derecho a la privacidad y consentimiento informado. Los autores han obtenido el consentimiento informado de los pacientes y/o sujetos referidos en el artículo. Este documento obra en poder del autor de correspondencia.

\section{Bibliografía}

1. Guevara-Ríos, E. La preeclampsia, problema de salud pública. Rev Peru Investig Materno Perinat. 2019;8:7-8.

2. De Kat AC, Hirst J, Woodward M, Kennedy S, Peters SA. Prediction models for preeclampsia: a systematic review. Pregnancy Hypertens. 2019;16:48-66.

3. Alegría-Guerrero R, Gonzales-Medina C. Evaluación diagnóstica de un nomograma de predicción de preeclampsia. Rev Peru Investig Materno Perinat. 2019;7:29-47.

4. Benites-Zapata VA, Lozada-Urbano M, Urrunaga-Pastor D, Márquez-Bobadilla E, Moncada-Mapelli E, Mezones-Holguín E. Factores asociados a la no utilización de los servicios formales de prestación en salud en la población peruana: análisis de la encuesta nacional de hogares (ENAHO) 2015. Rev Peru Med Exp Salud Pública. 2017;34:478-84.

5. Guerrero-Rosa A. Factores asociados a preeclampsia en gestantes atendidas en un hospital peruano, 2019. Revista Internacional de Salud Materno Fetal. 2019;4:27-34.

6. Díaz Pérez A, Roca-Pérez A, Oñate-Díaz G, Castro-Gil P, Navarro-Quiroz E. Interacción dinámica de factores de riesgo epidemiológicos presentes en los trastornos hipertensivos del embarazo: un estudio piloto. Salud (Barranquilla). 2017;33:27-38.

7. Brown MA, Magee LA, Kenny LC, Karumanchi SA, McCarthy FP, Saito S, et al. The hypertensive disorders of pregnancy: ISSHP classification, diagnosis and management recommendations for international practice. Pregnancy Hypertens. 2018;13:291-310.

8. Pérez-Bojurquez O, Lara-Ávila L, Gonz A, de Jess Peraza-Garay F, Ortiz FM. Valores de referencia normales para el índice de pulsatilidad de arterias uterinas en el primer y segundo trimestre del embarazo. Edu. mx. (Consultado el 21 de abril de 2021.) Disponible en: http://hospital. uas.edu.mx/revmeduas/pdf/v5/n1/pulsatilidad.pdf 
9. Mayrink J, Costa ML, Cecatti JG. Preeclampsia in 2018: revisiting concepts, physiopathology, and prediction. Sci World J. 2018;18:1-9.

10. Meertens LJE, Scheepers HCJ, van Kuijk SMJ, Aardenburg R, van Dooren IMA, Langenveld J, et al. External validation and clinical usefulness of first trimester prediction models for the risk of preeclampsia: a prospective cohort study. Fetal Diagn Ther. 2019;45:381-93.

11. The American College of Obstetricians and Gynecologist. Hypertension in pregnancy: executive summary. Obstet Gynecol. 2013;122:1122-31.

12. ACOG Committee Opinion. Low-dose aspirin use during pregnancy. Obstet Gynecol. 2018;132:e44-52.

13. Guevara Ríos E, Pérez Aliaga C, De la Peña Meniz W Limay Ríos O, Meza Santibáñez L, Ching Ganoza S, et al. Prevención y manejo clínico de pre eclampsia y eclampsia: guía de práctica clínica basada en evidencias del Instituto de Referencia Nacional en Salud Materna del Perú. An Fac Med. 2019;80:243-9.

14. Guevara E, Gonzales-Medina C. Factores de riesgo de preeclampsia una actualización desde la medicina basada en evidencias. Rev Peru Investig Matern Perinat. 2019;8:30-5.

15. Checya-Segura J, Moquillaza-Alcántara VH. Factores asociados con preeclampsia severa en pacientes atendidas en dos hospitales de Huánuco, Perú. Ginecol Obstet Mex. 2019;87:295-301.

16. Direkvand-Moghadam A, Khosravi A, Sayehmiri K. Predictive factors for preeclampsia in pregnant women: a receiver operation character approach. Arch Med Sci. 2013;4:684-9.

17. Quan L-M, Xu Q-L, Zhang G-Q, Wu L-L, Xu H. An analysis of the risk factors of preeclampsia and prediction based on combined biochemical indexes. Kaohsiung J Med Sci. 2018;34:109-12.

18. Benites-Cóndor Y, Bazán-Ruiz S, Valladares-Garrido D. Factores asociados al desarrollo de preeclampsia en un hospital de Piura, Perú. $\mathrm{Cl}$ MEL. 2011;16:77-82.

19. Morales Ruiz C. Factores de riesgo asociados a preeclampsia en el Hospital Nacional Daniel Alcides Carrión. Callao. Abril a junio de 2010. Rev Peru Epidemiol. 2011;15:97-101.

20. Tan MY, Syngelaki A, Poon LC, Rolnik DL, O'Gorman N, Delgado JL, et al. Screening for pre-eclampsia by maternal factors and biomarkers at 11-13 weeks' gestation. Ultrasound Obstet Gynecol. 2018;52:186-95.

21. Reyna-Villasmil E, Mayner-Tresol G, Herrera-Moya P, Briceño-Pérez C. Marcadores clínicos, biofísicos y bioquímicos para la predicción de preeclampsia. Rev Peru Ginecol Obstet. 2017;63:227-33.

22. Kafkaslı A, Türkçüoğlu I, Turhan U. Maternal, fetal and perinatal characteristics of preeclampsia cases with and without abnormalities in uterine artery Doppler indexes. J Matern Fetal Neonatal Med. 2013;26:936-40.
23. Bolin M, Wikström A-K, Wiberg-Itzel E, Olsson A-K, Ringvall M, Sundström-Poromaa I, et al. Prediction of preeclampsia by combining serum histidine-rich glycoprotein and uterine artery Doppler. Am J Hypertens. 2012:25:1305-10.

24. Lubis MP, Hariman H, Lumbanraja SN, Bachtiar A. The role of placental growth factor, soluble endoglin, and uterine artery diastolic notch to predict the early onset of preeclampsia. Open Access Maced J Med Sci. 2019;7:1153-9.

25. Abdel Razik M, Mostafa A, Taha S, Salah A. Combined Doppler ultrasound and platelet indices for prediction of preeclampsia in high-risk pregnancies. J Matern Fetal Neonatal Med. 2019;32:412832.

26. The American College of Obstetricians and Gynecologists. ACOG Practice Bulletin No. 202: Gestational hypertension and preeclampsia. Obstet Gynecol. 2019;133:e1-25.

27. Irfan KA, Fathima SM, Iqbal Y, Naseeruddin M. Role of uterine artery Doppler in 11-14 weeks scan as a predictor of preeclampsia. Ann Int Med Dent Res. 2017;4:8-11.

28. Espinoza Villena JS. Elaboración y aplicación de un normograma para predecir el desarrollo de preeclampsia en gestantes con factores de riesgo. [Tesis de grado]. Universidad Nacional de Trujillo; 2013. Disponible en: http://dspace.unitru.edu.pe/handle/UNITRU/253

29. Goetzinger KR, Tuuli MG, Cahill AG, Macones GA, Odibo AO. Development and validation of a risk factor scoring system for first-trimester prediction of preeclampsia. Am J Perinatol. 2014;31:1049-56.

30. Díaz Cobos D, Laparte C, Ruiz-Zambrana A, Alcázar JL. Screening de primer trimestre de preeclampsia tardía en gestantes de bajo riesgo en un centro de bajo volumen obstétrico: validación externa de un modelo predictivo. An Sist Sanit Navarra. 2015;38:387-96

31. Alzamora Sánchez A, Ponce Martos D. Factores asociados a la accesibilidad de los servicios de salud en pobladores del Asentamiento Humano San Francisco de Ñaña, 2020. [Tesis de grado]. Universidad Peruana Unión; 2020. Disponible en: http://repositorio.upeu.edu.pe/handle/ UPEU/3021

32. Alcalde-Rabanal J, Lazo-Gonzales O, Macias N, Contreras-Ochoa C, Espinosa Henao O. Sistema de salud de Perú: situación actual, desafíos y perspectivas. Revista Internacional de Salud Materno Fetal. 2019;4:8-18.

33. Stefańska K, Zieliński M, Zamkowska D, Adamski P, Jassem-Bobowicz J, Piekarska K, et al. Comparisons of dipstick test, urine protein-to-creatine ratio, and total protein measurement for the diagnosis of preeclampsia. Int J Environ Res Public Health. 2020;17:4195. 